\title{
Energy Efficiency Trade-Off Between Duty-Cycling and Wake-Up Radio Techniques in loT Networks
}

\author{
Adam Kozłowski ${ }^{1}$ (D) Janusz Sosnowski ${ }^{1}$ (D) \\ Published online: 26 April 2019 \\ (c) The Author(s) 2019
}

\begin{abstract}
Energy consumption has become dominant issue for wireless internet of things (IoT) networks with battery-powered nodes. The prevailing mechanism allowing to reduce energy consumption is duty-cycling. In this technique the node sleeps most of the time and wakes up only at selected moments to extend the lifespan of nodes up to 5-10 years. Unfortunately, the scheduled duty-cycling technique is always a trade-off between energy consumption and delay in delivering data to the target node. The delay problem can be alleviated with an additional wake-up radio (WuR) channel. In the paper we present original power consumption models for various duty-cycling schemes. They are the basis for checking whether WuR approach is competitive with scheduled duty-cycling techniques. We determine the maximum energy level that an additional wake-up radio can consume to become a reasonable alternative of widely used duty-cycling techniques for typical IoT networks.
\end{abstract}

Keywords Duty-cycling · Wake-up radio (WuR) - Energy consumption · Wireless sensor networks (WSNs) $\cdot$ Internet of things (IoT)

\section{Introduction}

The current trend of production process automation and data exchange between various devices, called Industry 4.0, introduces the so-called "smart factory" in which cyber-physical systems (CBSs) monitor the physical processes of the factory and make decentralized decisions. CBSs interact with each other and with humans in real time over internet of things (IoT) networks [1]. The efficiency of wireless sensor networks (WSN) is of great importance for the development of this concept. Typically, a WSN consists of a large number of lowcost and battery-powered nodes with limited functional resources. Usually, WSN nodes are deployed in a hostile and unattended environment, making battery replacement impractical.

Adam Kozłowski

a.kozlowski@ii.pw.edu.pl

Janusz Sosnowski

j.sosnowski@ii.pw.edu.pl

1 Faculty of Electronics and Information Technology, Institute of Computer Science, Warsaw University of Technology, Nowowiejska 15/19, 00-665 Warsaw, Poland 
Therefore, energy saving is the main concern for the design of an effective WSN [2, 3]. In the early stages, WSN network research focused mainly on data acquisition applications such as smart-grids or environmental and agriculture monitoring with low-rate delay tolerant schemes. Currently, WSN applications cover a wide range of solutions starting from industrial automation, through health-care, to automation of transport and multimedia $[4,5]$. These applications are known as delay-sensitive applications in which time issues should be taken into account. Therefore, node-to-node and end-to-end delay is the second main concern in WSN. Moreover, ensuring a predefined delay is challenging.

Currently, used radio transmitters consume approximately the same amount of energy when they receive data and when they listen to the channel waiting for upcoming transmissions (idle listening). Hence, to reduce energy consumption the WSN nodes switch the radio between active and sleep modes. In the active mode a node can receive and transmit packets, while in the sleep mode it completely turns off its radio to save energy. Since frames cannot be exchanged unless both the transmitter and the receiver are active, the node must either be aware of its neighbors' wake-up time or be able to wake up its neighbor node. The common technique used in WSN is duty-cycling, when the node sleeps most of the time and wakes up periodically only at scheduled moments. These moments are known to the transmitter which must wait a certain time for waking up its neighbor to exchange data. This issue has a direct and negative impact on the latency of the network. The scheduled duty-cycling technique is always a trade-off between energy consumption and delay in delivering the frame to the target node.

The delay problem can be alleviated by an additional wake-up radio (WuR) [6]. The idea is to use an additional receiver with such a low power consumption that it can be active all the time. A node will be woken up only on demand, which decreases communication delay and which is important in time-critical applications.

In the paper, we analyze various techniques used in the framework of the duty-cycling mechanism. We have found that an important issue is to compare WuR with other dutycycling techniques by evaluating the energy consumption directly related to the used dutycycling approach. This issue is neglected in the literature. In the article, we are looking for the answer to the question: "How much energy can consume WuR to be able to compete with other duty-cycling techniques?" The methodology for determining the energy consumption proposed in the paper takes into account only those elements of the generalized MAC protocol that are directly related to the used duty-cycling technique. The presented approach is universal and disregards implementations of the MAC protocol. These implementations are always addressed to a specific application class due to the inherent compromise between energy consumption and latency. Over the years, various MAC protocols have been created, each aimed at improving specific parameters in a very narrow view, e.g. energy consumption $[3,7]$, node-to-node and end-to-end latency [8, 9], network throughput [10], radio channel occupation [11, 12], packet delivery ratio [13]. The multiplicity of these protocols and the lack of their uniqueness make comparison hard. The approach based on their generalization applied in the proposed methodology ensures an effective comparison. Moreover, in recent years, a self-adaptive MAC protocols for WSN have been proposed [14]. The presented methodology can be effectively used in these protocols to find an optimum trade-off between various MAC layer parameters in order to find an optimum energy consumption level [15].

In the paper, we are referring to our previous studies [16] on energy consumption measurements done for nodes with modern Texas Instruments CC1310 chip. Based on these measurements, we have developed original and detailed power consumption models for various duty-cycling schemes. In particular, they include power cost related to clockdrift (problem neglected in the literature). The created models allow us to determine the 
maximum energy level that the wake-up radio can consume to become a good alternative solution for typical IoT networks.

The remainder of the paper is organized as follows. Section 2 outlines the existing communication schemes. It describes basic features and the power consumption optimization problem for various duty-cycling techniques and the wake-up radio approach. Section 3 presents the proposed network synchronization scheme, which assures energy savings. The original power consumption models are presented in Sect. 4. Section 5 comprises a comparative analysis of power consumption for five typical IoT networks. Findings of the study are listed in Sect. 6 and finally, conclusions are given in Sect. 7.

\section{Existing Communication Schemes}

In many applications of wireless sensor networks (WSN) the critical problem is efficient utilization of battery power to extend network lifetime [2]. Most papers in the literature concentrate on energy efficient communication protocols at the network level. These protocols take into account communication model, network structure and topology, reliable routing schemes [17-21]. An important issue is also detailed energy consumption analysis at the network node level (neglected in the literature) which is the goal of our studies. Moreover, it provides additional information for protocol optimization.

Over the years, a large number of MAC-layer protocols have been developed for use in WSN, e.g. B-MAC [7], A-MAC [22], RI-MAC [23], X-MAC [24]. Many studies in the literature compare the characteristics of these protocols focusing on a specific group. Doudou et al. [9] have reviewed asynchronous MAC protocols dedicated for time critical WSNs. Carrano et al. [25] have created a survey of duty-cycling mechanisms, dividing them into: synchronous, asynchronous and semi-synchronous. Huang et al. [26] have presented the evolution of MAC protocols over the years.

The technologies used in WuR and their parameters have changed in recent years. In particular, parameters such as sensitivity and energy consumption, have been significantly improved. Hence, WuR becomes attractive to be considered in WSNs. Blanckenstein et al. [27] have surveyed low-power transceivers and relate their characteristics to requirements for different application areas. Piyare et al. [28] have given a review of the research progress in wake-up radio (WuR) hardware and relevant networking software. In the sequel we discuss basic features of the cited WSN protocols. In particular, we focus on power consumption and transmission latency problems.

\subsection{Duty Cycle Schemes}

Transceivers used in WSN nodes usually employ FSK or PSK modulation variants. Despite significant progress in reducing energy consumption, the radio unit consumes typically $15-50 \mathrm{~mW}$ while listening to the radio channel. Assuming that the node is constantly listening for the upcoming transmission $(5 \mathrm{~mA})$ and that it is powered by $2 x \mathrm{xAA}$ batteries (about $750 \mathrm{mAh}$ ), the lifespan of such a node is only $150 \mathrm{~h}$, which is definitely below expectations. When designing WSN consisting of nodes with limited resources and battery-powered, we expect maintenance-free operation for at least 5 years. Therefore, the node must temporarily turn off its radio in order to reduce energy consumption and ensure adequate lifespan. The technique in which the nodes go periodically into sleep and wake up only for a short period is called duty-cycling. The most popular is a scheduled 
duty-cycling, in which nodes wake up at the scheduled time, so the transmission can take place only when the active time arrives. Another approach is presented by the on demand technique, which is based on the idea that the node can be awakened when necessary. It usually relies on another communication channel. The proposed duty-cycling taxonomy, which includes on demand wake-up radio approach is shown in Fig. 1.

The main problem of using the scheduled duty-cycling technique is the delay in the frame delivery to the target node and as a result end-to-end delay. Moreover, choosing the optimal time between subsequent awakenings is always a compromise between energy consumption and delay in the frame delivery. Ensuring communication between nodes, that are inactive most of the time, requires an additional energy related to the following issues:

Overhearing The node receives a transmission that is not intended (or no longer useful) for it.

Over-emitting The node sends data while the destination node is not ready to receive it. This does not happen with synchronous protocols, but only with asynchronous ones.

Idle-listening The node listens when other nodes do not send.

Synchronization Nodes send sync messages to ensure mutual synchronization. This applies only to synchronous duty-cycle.

Depending on how a sender joins its target receiver, scheduled duty-cycling technique can be synchronous or asynchronous. In the synchronous approach, neighboring nodes are synchronized to wake up at the same time. The synchronization can take place using internal or external synchronization sources, see Sect. 3. In internal synchronization, nodes periodically exchange synchronization messages, which requires additional energy. While using external synchronization, the number of possible synchronization sources is very limited and the node requires more complicated hardware. On the other hand, in the asynchronous approach node clocks are independent which eliminates synchronization overhead but can significantly increase the amount of energy needed to meet the active period of another node. Moreover, the synchronous protocols are heavily affected by clock drift [29], while asynchronous protocols do not experience this effect. In asynchronous rendezvous, the node has no knowledge of awakening time of the neighboring node and therefore must turn on its radio and remain active to ensure communication when the neighbor is active. The node can wait for an adjacent node either by broadcasting or listening depending on the technique used.

Initially, MAC protocols based on asynchronous rendezvous used sender-initiated low power listening (LPL) to reduce energy consumption on the receiver side. B-MAC [7] is the first LPL protocol in which a sender starts transmitting a long preamble to meet receiver.

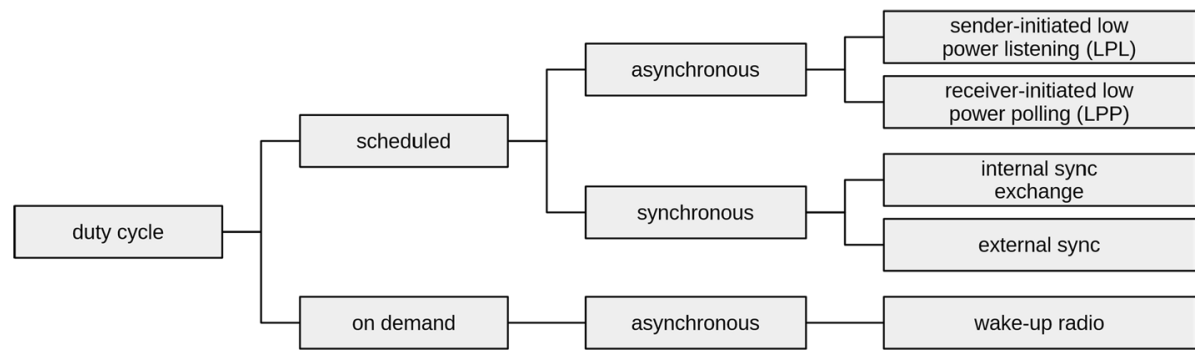

Fig. 1 The taxonomy of duty-cycling techniques 
The over-emitting and the overhearing effects are significant drawbacks in their practical use. The communication channel is occupied by the transmitter during the entire preamble phase, making it impossible for other senders to communicate effectively. X-MAC [24] and other subsequent LPL based protocols limit these effects. The over-emitting effect reduction is possible by applying a strobed preamble (multiple short preambles) and by sending an early acknowledgment (ACK). Nevertheless, this effect still remains the main limitation. The overhearing effect can be reduced using short preambles comprising target node ID so that the non-target receiver can quickly go back to sleep. In Fig. 2 (left), TX1 node initiates rendezvous by sending a strobed preamble (sequence of ID1s). The channel is occupied during the whole handshake procedure, hence TX2 node is not able to start transmission. The RX1 node to which the TX1 transmission is addressed sends a confirmation (ACK) as soon as it receives the preamble. TX1 starts the data transmission after receiving the confirmation. Due to the high channel occupancy, the use of LPL is limited only to applications in which the traffic load is low and the nodes transmit infrequently. According to EU harmonized regulations ETSI EN 300 220-2 (range $25 \mathrm{MHz}-1 \mathrm{GHz}$ ), the channel can be occupied for only a fraction of time or a polite spectrum access has to be used. Similar regulations can be found in other countries, therefore LPL cannot be widely used in practical applications.

Another asynchronous technique is the receiver-initiated low power pooling (LPP). The advantage of the LPP design is that the channel is free for use before the target receiver is ready to receive. The idea of shifting communication initiation from the sender side to the receiver side is early presented in RICER [30]. LPP for WSN was introduced in RI-MAC [23] and extended in A-MAC [22]. Differently from the LPL, the sender instead of transmitting a preamble, waits for a beacon from the receiver and transmits the frame only after its reception. This substitutes the periodic beacons for the strobe preamble used in LPL, with the advantage, that the receiver beacon does not occupy the medium for as long as the sender preamble. In Fig. 2 (right), TX1 node is going to send data, therefore it listens to the channel while waiting for the beacon (ID1) from the target receiver. Both RX1 and RX2 nodes send beacons as identification messages but only if the channel is available (2nd ID1). As soon as TX1 receives beacon from the target node, it starts the current data transmission.

Moreover, it is worth noting that over the years, many protocols based on the LPL or LPP techniques have been tailored to specific applications [26]. Numerous proposals use an adaptive approach, dynamically adjusting the node wake up time based on various network parameters. All these variations share a common problem which is a tradeoff between energy consumption and delay. The use of an on-demand approach eliminates this problem, but requires the assignment and maintenance of an additional communication channel.


Fig. 2 The LPL (left) and LPP (right) asynchronous rendezvous 


\subsection{Wake-Up Radio Approach}

The use of low-power wake-up radio (WuR) can significantly reduce the overall power consumption of the system. However, more importantly, it allows to reduce the delay in frame delivery to the target node, which is particularly important in delay-sensitive applications especially in Industry 4.0. The idea is to use an additional receiver with such a low power consumption that it will be possible to keep it active all the time. The main receiver will not be woken up periodically to listen to the channel, but only on demand in order to receive actual data. The energy consumption of $\mathrm{WuR}$ when compared to the main radio can be reduced by: energy harvesting, modulation technique, limiting sensitivity, limiting bitrate or using lower frequencies for wake-up triggering [31]. WuR is not a new idea but in recent years it gains more interest. In 2017, 802.11ba standard task group (TG) was created as part of IEEE's standard: IEEE Std 802.11 (i.e., Wi-Fi), to develop the WuR standard for wireless local area networks. This standard is expected to be approved in 2020, which will increase the availability of commercial WuR solutions. Ready-to-use WuR devices will help researchers to study practical applications, so new interesting results will emerge.

The WuR hardware design can be classified into active and passive approaches. The active WuR requires energy from external source e.g. battery. In contrast, the passive one harvests and powers the wake-up circuitry entirely from the RF signal, so it does not require energy from battery at all. Although passive WuRs are energy efficient and offer extended lifetimes, they have a much shorter operating range than active WuRs, typically only a few meters. Moreover, the process of accumulating energy also delays the wake-up of the main unit, affecting network performance by increasing latency. The active WuR addresses the constraints of passive one by increasing sensitivity and providing longer range with reasonable power consumption.

Over the years, various hardware solutions have been proposed and studied. There are several surveys $[27,28]$ that review presented proposals in terms of key characteristics of WuR technology such as power consumption, sensitivity and data rates. Most of the concepts use amplitude modulation in its binary form on-off-keying (OOK), only a few use a more complex one. OOK has the advantage of overall implementation simplicity which can be transferred into energy efficiency. Available solutions work in the subGHz or $2.4 \mathrm{GHz}$ range.

Nevertheless, most of the WuR designers have opted to shift from high frequency to sub-GHz as an operating frequency for wake-up receivers. One of the reasons is that at higher frequencies the attenuation rate also increases, i.e., the $2.4 \mathrm{GHz}$ signal fades faster than a sub-GHz signal. According to the Friis equation [32], the path loss at $2.4 \mathrm{GHz}$ is $8.83 \mathrm{~dB}$ higher than at $868 \mathrm{MHz}$ resulting into 2.76 times longer range for $868 \mathrm{MHz}$ transceivers. The node with the main transceiver operating at $2.4 \mathrm{GHz}$ with high sensitivity, e.g. $110 \mathrm{dBm}$ can use WuR with lower sensitivity, e.g. $80 \mathrm{dBm}$, working at lower frequency to compensate power link budget. Moreover, lowering the bit rate allows to increase the signal-to-noise ratio (SNR) margin and consequently the range, unfortunately it extends the time (increases the energy consumption) needed to transmit a specific awakening sequence.

Besides the need for higher power for the same link budget, $2.4 \mathrm{GHz}$ frequency band is more prone to interference due to spectrum crunch and devices such as Wi-Fi and Bluetooth operating in the same band. Sub-GHz ISM bands are mostly used for proprietary low duty-cycling links and are less likely to interfere with each other. This means easier transmission and fewer communication retries, which is more efficient and saves battery power.

An important issue is to compare power consumption of WuR networks versus dutycycling schemes. Dealing with this problem we have developed power consumption models 
taking into account various parameters characterizing communication processes (Sect. 4). In these models we include also clock drift power cost which is neglected in the literature (Sect. 3). The analytical considerations have been used to compare WuR approach with duty-cycling schemes within a representative class of IoT networks (Sect. 5).

\subsection{Synchronization Problem}

Precise timing is especially important in synchronous duty-cycling. Nodes communicating with each other must ensure synchronization between the start of transmission by one node and listening by the other one. Due to the limited accuracy of the clocks in the nodes, the network must provide a mechanism to synchronize the clocks in neighboring nodes [33]. Broadcasting beacons is a commonly used mechanism to assure such synchronization. In this technique, one node, most often a coordinator, sends a beacon frame, which is a reference time point for neighboring nodes that synchronize their clocks. Depending on whether the synchronization takes place in the whole network, its part, or concerns only neighboring nodes, the frames are sent in a cascade within a given area or independently. Synchronization takes place periodically, with the period related to the maximum value of node clock inaccuracy. Adaptive solutions are also used, which determine the synchronization period for a group of nodes based on the actual rather than maximum clock inaccuracy values [34]. The synchronization can also rely on a reliable clock source that is external to the wireless node. This could be a GPS receiver [35], the FM RDS signal [36] or national LF radio time signals, such as DCF77 [37] which is available in Europe. However, these techniques are not widely used, mainly due to the complicated design of the receiver and additional energy consumption (as in the case of GPS) or limited coverage (as in the case of DCF77).

The choice of a real-time clock (RTC) oscillator operating in the sleep mode is always a compromise between energy consumption and accuracy, which creates an additional issue in synchronization. Let us consider nodes based on popular microcontroller chip CC1310. This chip has low-speed (LF) and high-speed (HF) clocks. LF clock is also available in STANDBY mode. The RTC is clocked from the LF 32-kHz RC oscillator (RCOSC_LF) or crystal oscillator (XOSC_HF). The RC oscillator consumes less energy but also has lower accuracy. The frequency accuracy of the Real Time Clock (RTC) is not directly dependent on the frequency accuracy of the $32-\mathrm{kHz}$ RC Oscillator. The RTC can be calibrated periodically to accuracy within $\pm 500 \mathrm{ppm}$ of $32.768 \mathrm{kHz}$ by measuring the frequency error of RCOSC_LF relative to XOSC_HF and compensating the RTC tick speed. The accuracy of the RTC clocked from the crystal oscillator depends mainly on the crystal frequency tolerance and usually varies from 10 to $50 \mathrm{ppm}$. Additional software based calibration can be used to reduce clock drift caused by temperature changes.

Due to the clock inaccuracy, the nodes must take into account the maximum error and wake up in advance to enter the listening mode which results in idle listening. When synchronizing nodes using beacon frames, extending the time between successive beacons reduces energy consumption for exchanging frames, while on the other hand, it increases the energy consumption due to idle listening while waiting for data transmission. 


\section{The Proposed Synchronization Scheme}

Analyzing the problem stated in the Sect. 2.3 we have noticed, that the time necessary to mitigate the clock inaccuracy while receiving data, depends on the time that has elapsed since the last synchronization. Further, we present a network communication scheme where nodes are synchronized using beacons. Based on this scheme, we derive an equation for calculating the energy consumption of synchronization mechanism. The presented approach takes into account the time that has elapsed since the last received synchronization beacon. In order to analyze the effect of clock inaccuracy on synchronization, we consider a cluster consisting of $\mathrm{N}$ nodes. The analyzed network consists of clusters where every cluster has its own and independent synchronization policy. Each node listens to the channel periodically and it waits for data for a predetermined time (time slot), according to the following scheme:

1. The cluster coordinator starts broadcasting the beacon frame. The nodes that have received the frame join the cluster by selecting the sender as the parent node and then broadcasting their own beacon. Nodes that do not have a parent node and have received beacon, select the sender as the parent node and broadcast their own beacon. In steady state, for $\mathrm{N} \gg 1$ each node receives beacon from the parent node and broadcasts its own beacon. The time between sending adjacent beacons is $\mathrm{T}_{\text {beacon }}$.

2. Every node joined to the cluster starts listening to upcoming data periodically, consider that the time between adjacent time slots is $T_{\text {data }}$.

3. Let $\Theta$ (expressed in ppm) is maximum RTC inaccuracy for the entire cluster.

4. The idle listening time while waiting for upcoming beacon is $t_{I L \_ \text {beacon }}=2 \cdot \Theta \cdot T_{\text {beacon }}$.

5. The idle listening time while waiting for upcoming data is $t_{I L_{-} \text {data }}=4 \cdot \Theta \cdot \tau$ where $\tau$ is the time since the last synchronization $\tau \in\left(0, T_{\text {beacon }}\right)$. Assuming $\mathrm{T}_{\text {data }}<\mathrm{T}_{\text {beacon, }}$, the average value of $\tau$ is $T_{\text {beacon }} / 2$, see Fig. 3 for details.

6. The total energy consumed while transmitting and receiving single beacon (without idle-listening time) is defined as $\mathrm{E}_{\mathrm{T}_{-} \text {beacon }}$ and $\mathrm{E}_{\mathrm{R}_{-} \text {beacon }}$ respectively. Power dissipated in radio idle listening mode is defined as $P_{I L}$.

Figure 3 presents the instantaneous power consumption as a function of time for two cluster nodes $\mathrm{N} 1$ and $\mathrm{N} 2$. N1 sends the periodic beacon consuming $\mathrm{E}_{\mathrm{T} \text { _beacon }}$ energy while $\mathrm{N} 2$ receives it consuming $\mathrm{E}_{\mathrm{R} \_ \text {beacon }}$. Beacons are sent with the $\mathrm{T}_{\text {beacon }}$ period. There are several time slots between adjacent beacons when nodes are waiting for

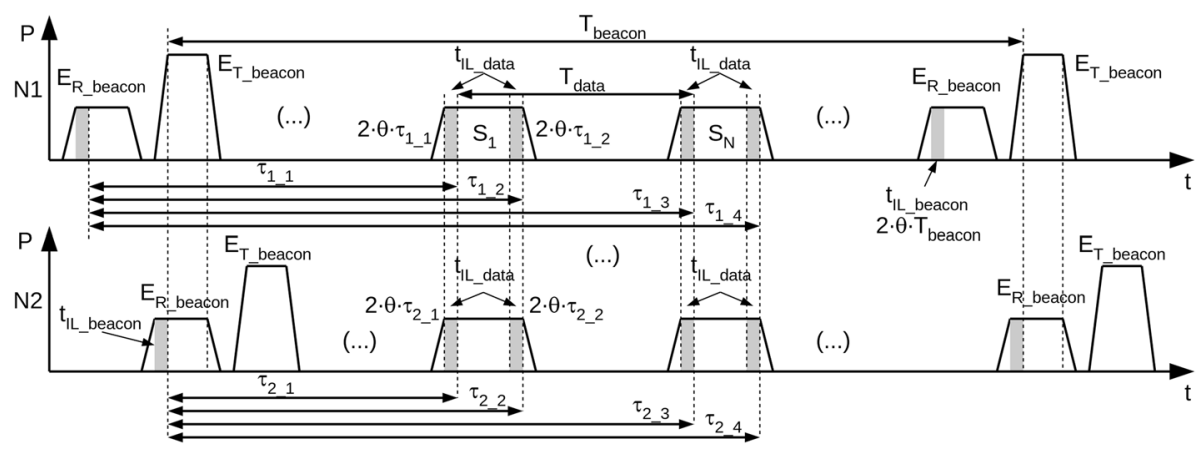

Fig. 3 Beaconing and idle-listening due to clock drift 
upcoming data $\left(\mathrm{S}_{1}, \ldots, \mathrm{S}_{\mathrm{N}}\right)$. These slots are cyclic with the $\mathrm{T}_{\text {data }}$ period. The nominal time slot duration is fixed and the dashed area denotes energy consumed for the idle-listening related to the clock-drift. We can notice that the duration of this dashed phase is directly proportional to the time since the last synchronization (last beacon reception). The idle listening time when receiving beacon $t_{I L_{-} \text {beacon }}$ is constant and proportional to the $\mathrm{T}_{\text {beacon }}$ period. However, the idle listening time when waiting for data $t_{I L_{-} \text {data }}$ can be optimally adjusted. This time is short when the slot appears directly after the beacon reception and it extends in the subsequent time slots. According to this, nodes have to adapt $t_{I L \_ \text {data }}$ using both the $\Theta$ parameter and time since the last beacon reception. This $t_{I L \_ \text {data }}$ time is equal to $2 \cdot \Theta \cdot \tau_{1 \_1}+2 \cdot \Theta \cdot \tau_{1 \_2}$ for the S1 time slot in the N1 node, where $\tau_{1 \_1}, \tau_{12}$ represent time since the last synchronization. Therefore, to conserve energy, this adaptation of $t_{I L \_ \text {data }}$ duration has to be implemented in practical applications.

According to a general model from [38], we have derived more detailed model of power dissipated due to synchronization in the node. It takes into account power losses related to synchronization offsets of beacons $t_{I L_{-} \text {beacon }}$ and data slots $t_{I L_{-} \text {data }}$

$$
P_{\text {sync }}=P_{T_{-} \text {beacon }}+P_{R_{-} \text {beacon }}+P_{\Theta_{-} \text {IdleListening }}
$$

where

$$
P_{T_{-} \text {beacon }}=\frac{E_{T_{-} \text {beacon }}}{T_{\text {beacon }}}
$$

is the power contribution due to transmission of periodic beacon

$$
P_{R_{\_} \text {beacon }}=\frac{\left(E_{R_{\_} \text {beacon }}+T_{\text {beacon }} \cdot 2 \cdot \Theta \cdot P_{I L}\right)}{T_{\text {beacon }}}
$$

is the power contribution due to receiving periodic beacons

$$
P_{\Theta \_ \text {IdleListening }}=\frac{T_{\text {beacon }} \cdot 2 \cdot \Theta \cdot P_{I L}}{T_{\text {data }}}
$$

is the power contribution due to idle listening caused by clock drift

Based on Eq. (1), the optimum $\mathrm{T}_{\text {beacon }}$ period can be found using the first derivative with respect to $T_{\text {beacon }}$ which have to be equal zero to find $P_{\text {sync }}$ minimum

$$
T_{\text {beacon }}=\sqrt{\frac{T_{\text {data }} \cdot\left(E_{T_{-} \text {beacon }}+E_{R_{-} \text {beacon }}\right)}{2 \cdot \Theta \cdot P_{I L}}} .
$$

The optimum $\mathrm{T}_{\text {beacon }}$ as a function of $\mathrm{T}_{\text {data }}$ for different clock inaccuracy is presented in Fig. 4. It results from Eq. (5).

\section{Energy Consumption Models}

To compare different communication techniques when nodes sleep most of the time, we should only consider energy that is lost by the mechanisms ensuring such communication $\left(\mathrm{P}_{\text {duty-cycle }}\right)$. Other elements, such as data transmission or message exchange, which are specific to MAC layer solutions, should be skipped. 

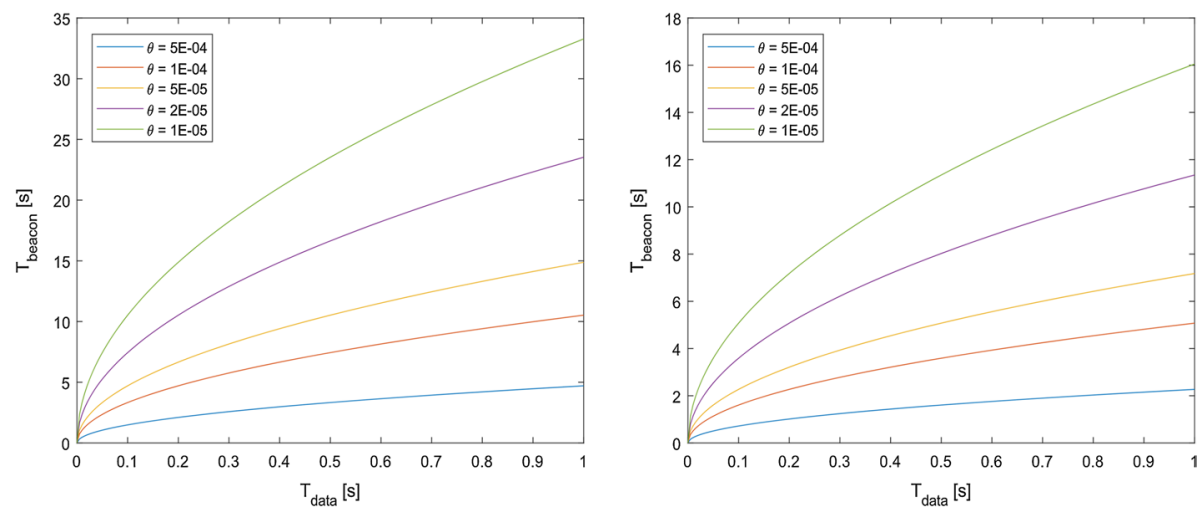

Fig. 4 Optimum $\mathrm{T}_{\text {beacon }}$ versus Tdata for CC1310 at $50 \mathrm{kBaud} / \mathrm{s}$ (left) and $400 \mathrm{kBaud} / \mathrm{s}$ (right)

Based on the measurements and earlier definitions in [16], we can determine the energy consumption while receiving and transmitting frames after waking the node. Let $l_{F}$ be the total frame length in bits, $R$ is the bit rate in bits per second and $t_{I L}$ is the time for idlelistening. The total energy for receiving $\left(\mathrm{E}_{\mathrm{RX} \_ \text {total }}\right)$ and transmitting $\left(\mathrm{E}_{\mathrm{TX} \_ \text {total }}\right)$ a frame can be expressed as a function of these variables, respectively:

$$
\begin{gathered}
E_{R X_{-} \text {total }}=f\left(l_{F}, R, t_{I L}\right)=E_{O N_{-} \text {total }}+E_{I N I T_{-} \mathrm{CMD}}+E_{I L}+E_{R X}+E_{R X_{-} \text {END_CMD }}+E_{O F F_{-} \text {total }} \\
E_{T X_{-} \text {total }}=f\left(l_{F}, R\right)=E_{O N_{-} \text {total }}+E_{I N I T_{-} \mathrm{CMD}}+E_{T X}+E_{T X_{-} \mathrm{END}_{-} \mathrm{CMD}}+E_{O F F_{-} \text {total }}
\end{gathered}
$$

where according to [16]:

- $\mathrm{E}_{\mathrm{ON} \_ \text {total }}$ is the total device energy consumption during the power-up phase. $\mathrm{E}_{\mathrm{OFF} \_ \text {total }}$ is the total energy consumption when going to deep sleep mode.

- $\mathrm{E}_{\text {INIT_CMD }}$ is the energy consumption during the initialization of the radio command. $\mathrm{E}_{\mathrm{RX} \_ \text {END_CMD }}$ and $\mathrm{E}_{\mathrm{TX} \_ \text {END_CMD }}$ are the energy consumption for completing the radio command for receiving and transmitting respectively.

- $\mathrm{E}_{\mathrm{RX}}$ and $\mathrm{E}_{\mathrm{TX}}$ are the energy consumption when receiving and transmitting data respectively. $\mathrm{E}_{\mathrm{IL}}$ is the idle-listening energy consumption.

In order to derive the energy consumption of duty cycle mechanism, consider a cluster consisting of $\mathrm{N}$ nodes. Each node has RTC with inaccuracy $\theta$ [ppm] and the maximum network RTC inaccuracy is $\Theta$ [ppm]. Assuming, single frame and single hop mode we can specify the probability of successful frame delivery as:

$$
p_{\text {suc }}=p_{\text {tr }} \cdot(1-B E R)^{l_{F}}
$$

where ptr is the probability to enter transmission stage (for contention based access) described in [39], $l_{F}$ is a total frame length in bits and BER is the bit error ratio.

Further, we present two schemes of the duty-cycle mechanism: synchronous and asynchronous one using the LPP technique. Based on the above-mentioned assumptions, we derive the formula for $\mathrm{P}_{\text {duty-cycle }}$ and we present its relationship with the key MAC layer parameters of the network. 


\subsection{Synchronous Duty Cycling}

The network uses a synchronous schedule duty-cycle mechanism without contention based access to the communication channel $\left(p_{t r}=1\right)$ where transceiver uses the channel during predetermined time slots.

1. Each node broadcasts and receives beacon. The time between sending adjacent beacons is $\mathrm{T}_{\text {beacon }}$.

2. Every node periodically listens for upcoming data. The time slot duration is $t_{\text {slot }}$. The time between adjacent slots is $\mathrm{T}_{\mathrm{s} \_ \text {data. }}$.

3. When a node misses the synchronization beacon it extends the idle listening time to cover additional clock-drift. Hence, taking into account $\mathrm{p}_{\text {suc }}(8)$, the expected value of the idle listening time can be rewritten as:

$$
t_{I L \_ \text {data }}=t_{I L \_ \text {beacon }}=p_{\text {suc }} \cdot \sum_{i=1}^{\infty}\left[\left(1-p_{\text {suc }}\right)^{i-1} \cdot 2 \cdot i \cdot T_{\text {beacon }} \cdot \Theta\right]=\frac{2 \cdot T_{\text {beacon }} \cdot \Theta}{p_{\text {suc }}}
$$

Based on Eq. (1), we can derive energy consumption for synchronous duty-cycle as:

$$
P_{\text {duty - cycle }}=P_{T_{-} \text {beacon }}+P_{R_{-} \text {beacon }}+P_{\text {Slot_tdleListening }}
$$

where

$$
\begin{gathered}
P_{T \_ \text {beacon }}=\frac{E_{T X \_ \text {total }}\left(l_{F \_ \text {beacon }}, R\right)}{T_{\text {beacon }}} \\
P_{R \_ \text {beacon }}=\frac{E_{R X \_ \text {total }}\left(l_{F \_ \text {beacon }}, R, t_{I L \_ \text {data }}\right)}{T_{\text {beacon }}}
\end{gathered}
$$

are defined according to Eqs. (2) and (3) using Eqs. (6) and (7) respectively. The beacon length in bits is $l_{F_{-} \text {beacon }}$

$$
P_{\text {Slot_IdleListening }}=\frac{E_{R X_{-} \text {total }}\left(0, R, t_{I L \_ \text {data }}+t_{\text {slot }}\right)}{T_{S \_ \text {data }}}
$$

is the power contribution due to idle listening when waiting for upcoming transmission. The $t_{\text {slot }}$ factor of this equation can be considered either as a part of duty-cycle itself and as a part of the actual data transmission.

The synchronous $\mathrm{P}_{\text {duty-cycle }}$ as a function of $\mathrm{T}_{\text {beacon }}$ for different $\mathrm{T}_{\mathrm{s}_{\text {data }}}$ is presented in Fig. 5. The calculations were made using typical parameters: $B E R=1 \mathrm{E}-4, \Theta=50 \mathrm{ppm}$. The beacon length has been assumed to be representative based on the beacon frame format in the IEEE 802.15.4 standard, hence $l_{F-\text { beacon }}=32 \mathrm{~B} \cdot 8$. The time slot has been adopted as relatively short to minimize its impact on the duty-cycle energy consumption, so $t_{\text {slot }}$ is equal to $10 \mathrm{~B}$ at a given bit rate. For other parameters similar plots can be obtained from given equations. 

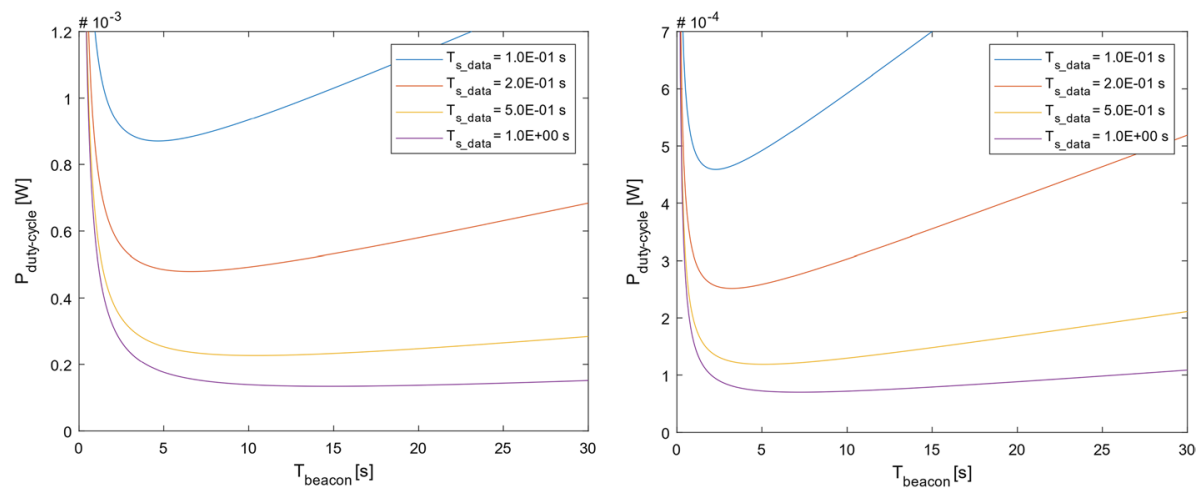

Fig. 5 The synchronous $P_{\text {duty-cycle }}$ versus $T_{\text {beacon }}$ for CC1310 at $50 \mathrm{kBaud} / \mathrm{s}$ (left) and $400 \mathrm{kBaud} / \mathrm{s}$ (right)

\subsection{Asynchronous LPP Duty Cycling}

The network uses a LPP asynchronous schedule duty-cycle mechanism, according to the following scheme:

1. Each node broadcasts beacon to indicate that is ready to receive data. The time between sending adjacent beacons is $\mathrm{T}_{\text {beacon. }}$. The beacon length in bits is $l_{F_{-} \text {beacon }}$ and time to send it at the given bit rate $\mathrm{R}$ is $t_{F_{-} \text {beacon }}=\frac{1}{R} \cdot l_{F_{-} \text {beacon. }}$. When the node finishes sending the beacon, it starts listening for arriving data for constant time $t_{I L_{-} \text {data }}$.

2. Every node sends data randomly, the time between adjacent samples is distributed according to the standard uniform distribution $U\left(0,2 \cdot T_{a_{-} \text {data }}\right)$ hence, the mean period is $T_{a_{-} \text {data }}$.

3. The node that wants to send data listens to the channel over time $t_{I L_{-} \text {beacon }} \in\left(t_{F_{-} \text {beacon }}, \quad t_{F_{-} \text {beacon }}+T_{\text {beacon }}+2 \cdot \Theta \cdot T_{\text {beacon }}\right)$ to catch the beacon of the target node. The nodes are decoupled and operate independently, so $\overline{t_{I L \_} \text {beacon }}=t_{F_{-} \text {beacon }}+T_{\text {beacon }} \cdot\left(\frac{1}{2}+\Theta\right)$ is the mean idle-listening time.

4. Nodes use contention to access channel, hence $p_{t r}<1$.

We can derive energy consumption when using LPP asynchronous duty-cycle as:

$$
P_{\text {duty - cycle }}=P_{T_{-} \text {beacon }}+P_{I L_{-} \text {data }}+P_{I L_{-} \text {beacon }}
$$

where

$$
P_{T_{-} \text {beacon }}=\frac{E_{T X_{-} \text {total }}\left(l_{F \_ \text {beacon }}, R\right)}{T_{\text {beacon }}}
$$

is the power contribution due to transmission of periodic beacon to indicate that the node is ready to receive data

$$
P_{I L \_ \text {data }}=\frac{E_{I L}\left(t_{I L \_ \text {data }}\right)}{T_{\text {beacon }}}
$$


is the power contribution due to idle listening for incoming data after the beacon was sent.

$$
P_{I L \_ \text {beacon }}=\frac{E_{I L}\left(t_{I L \_ \text {beacon }}\right)}{T_{a \_ \text {data }}}
$$

is the power contribution caused by listening to the channel in order to catch the beacon of the target node.

$\mathrm{P}_{\mathrm{IL} \text { beacon }}$ consists of only the idle listening energy, the other parts such as the energy necessary to power-up the node $\mathrm{E}_{\mathrm{ON} \_ \text {total }}$ and going to standby state $\mathrm{E}_{\mathrm{OFF} \_ \text {total }}$ are associated with data transmission and should not be included as a duty-cycle mechanism contribution. Based on Eq. (14), optimum $\mathrm{T}_{\text {beacon }}$ period can be found using the first derivative with respect to $\mathrm{T}_{\text {beacon }}$ which have to be equal zero to find $\mathrm{P}_{\text {duty-cycle }}$ minimum

$$
T_{\text {beacon }}=\sqrt{\frac{T_{a_{\_} \text {data }} \cdot\left[E_{T X_{-} \text {total }}\left(l_{F_{-} \text {beacon }}, R\right)+E_{I L}\left(t_{I L \_ \text {data }}\right)\right]}{(1 / 2+\Theta) \cdot P_{I L}}}
$$

The asynchronous LPP $\mathrm{P}_{\text {duty-cycle }}$ as a function of $\mathrm{T}_{\text {beacon }}$ for different $\mathrm{T}_{\mathrm{a} \_ \text {data }}$ is presented in Fig. 6. The calculations were made for typical parameters: $l_{F_{-} \text {beacon }}=16 B \cdot 8$, $\Theta=50 \mathrm{ppm}, \mathrm{t}_{\mathrm{IL} \_ \text {beacon }}$ as equivalent to $8 \mathrm{~B}$ (preamble $4 \mathrm{~B}+$ sync word $4 \mathrm{~B}$ ) at a given bit rate. The beacon length has been taken shorter than in the previous scenario, due to the skipped security part of a frame (without authentication). We believe that a "fake" beacon (with authenticity not proved) is much less problematic in this case than in the case of a synchronous technique.

The derived models can be used to effectively compare duty-cycling techniques without focusing on the specific implementation of the MAC protocol. The presented graphs are created assuming some illustrative parameters, however, other plots can be derived for other values if required. The presented approach based on the MAC generalization applied in the proposed methodology allows us to perform efficient comparisons. Furthermore, this methodology can be effectively used in adaptive MAC protocols to find an optimum tradeoff between various MAC layer parameters in order to find an optimum energy consumption level.
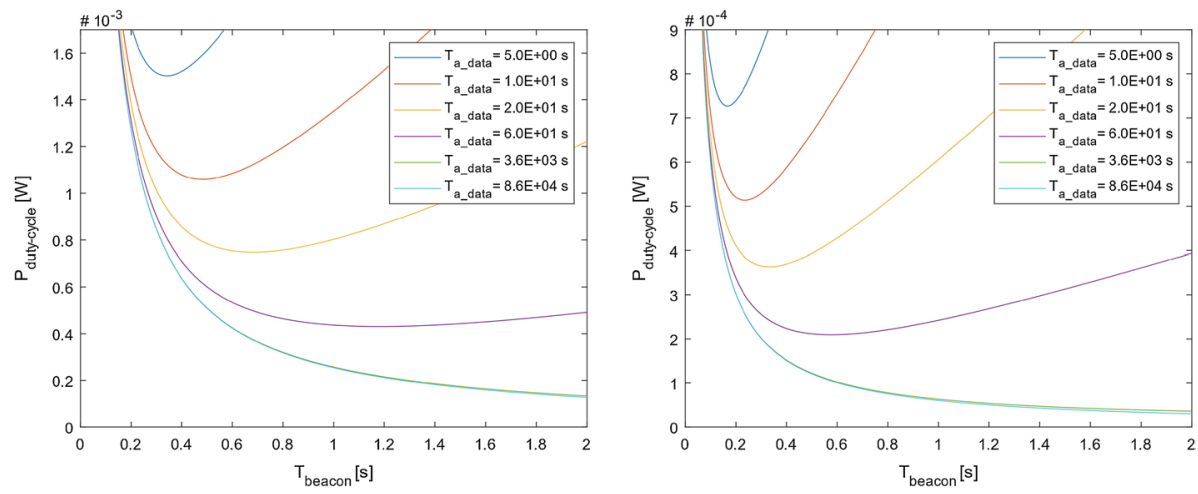

Fig. 6 The asynchronous LPP Pduty-cycle versus $\mathrm{T}_{\text {beacon }}$ for CC1310 at $50 \mathrm{kBaud} / \mathrm{s}$ (left) and $400 \mathrm{kBaud} / \mathrm{s}$ (right) 


\section{Comparative Analysis}

The derived power consumption models in Sect. 4, can be used in a comparative analysis of scheduled duty-cycling and WuR approaches. We perform this analysis for five representative network classes: industrial network, body area network (WBAN), smart metering, smart city, smart home. These network classes have been characterized with seven features: the maximum distance between nodes, the maximum node-to-node delay, the bit-rate range, the frequency of sending data, the size of sent data, node density, fault tolerance. These features are given successively in columns of Table 1. Depending on the network class (application scenario) the feature specifications differ. These specifications are not meant to be strict nor fixed limits for the shown network categories. Nevertheless, they can be considered as representative for these networks. They result from our practice and literature survey. All these features have a direct impact on the energy consumed by the duty-cycling mechanism. They allow us to point the applicability of WuR approach in the studied networks. Comparing power consumption in the considered network classes we use the derived formulas in Sect. 4 and appropriate values of the relevant parameters: $\Theta, R$, $\mathrm{T}_{\mathrm{s}_{\_} \text {data }}, \mathrm{t}_{\text {slot }}$ for the synchronous technique and $\Theta, \mathrm{R}, \mathrm{T}_{\text {beacon }}, \mathrm{T}_{\mathrm{a} \_ \text {data }}$ for the asynchronous one. The values of these parameters have been selected so as to assure similar functional features of the network for synchronous and asynchronous transmission. In the performed calculations, we assume the typical clock drift $\Theta=50 \mathrm{ppm}$ and bit rate (R) from the range given in the "bit rate" column in Table 1 . The period of listening time slots $\left(\mathrm{T}_{\mathrm{s} \_ \text {data }}\right)$ for the synchronous technique and the period of sending beacons $\left(\mathrm{T}_{\text {beacon }}\right)$ for the asynchronous one, were chosen so that the maximum node-to-node communication latency would meet the requirements indicated in the column "Delay" of Table 1 . The period of sending subsequent data samples $\left(\mathrm{T}_{\mathrm{a}_{\_} \text {data }}\right)$ for the asynchronous technique was assumed based on the general requirements from the "Data frequency" column in Table 1. Here, we assumed distinguishing values: $5 \mathrm{~s}, 10 \mathrm{~s}$ and $3600 \mathrm{~s}$ for medium, low and very low data frequency, respectively. The duration of listening time slot $\left(\mathrm{t}_{\text {slot }}\right)$ for synchronous technique was assumed as $10 \mathrm{~ms}$ - typical value for most network classes, except for smart home and smart metering where data frequency is low or very low, respectively. These parameters are listed in Tables 2, 3, 4 and 5, for each class and communication technique.

Employing proposed parameters and using the formulas (10) and (14) given in Sect. 4, along with formulas specifying included terms, we calculate a representative value of energy consumed due to the duty-cycling mechanism for synchronous and asynchronous LPP techniques, respectively. Using this approach $\mathrm{P}_{\text {duty_cycle }}$ can also be calculated for other parameters and for other network classes.

Body area network is the most promising application of WuR due to the short distance between adjacent nodes, which for many applications does not exceed $1 \mathrm{~m}$. The delay in this type of the network is not critical and allows using both synchronous and asynchronous mechanisms. The expected transmission speed is relatively high and requires a carrier frequency of at least $2.4 \mathrm{GHz}$. According to the derived equations, we can find the level of $\mathrm{P}_{\text {duty-cycle. }}$. In Table 2, we present values of the assumed parameters and the calculated energy level $\mathrm{P}_{\text {duty-cycle }}$ for both mechanisms. It is worth noting that in some applications a very short distance between adjacent nodes gives the possibility to use passive WuR, which significantly reduces energy consumption.

In our calculations, we base on previously performed measurements [16] made for the $\mathrm{CC} 1310$ (TI) chip, which works only in the $<1 \mathrm{GHz}$ range. For the purposes of presented calculations for the $2.4 \mathrm{GHz}$ range, we introduce a correction in the above mentioned 


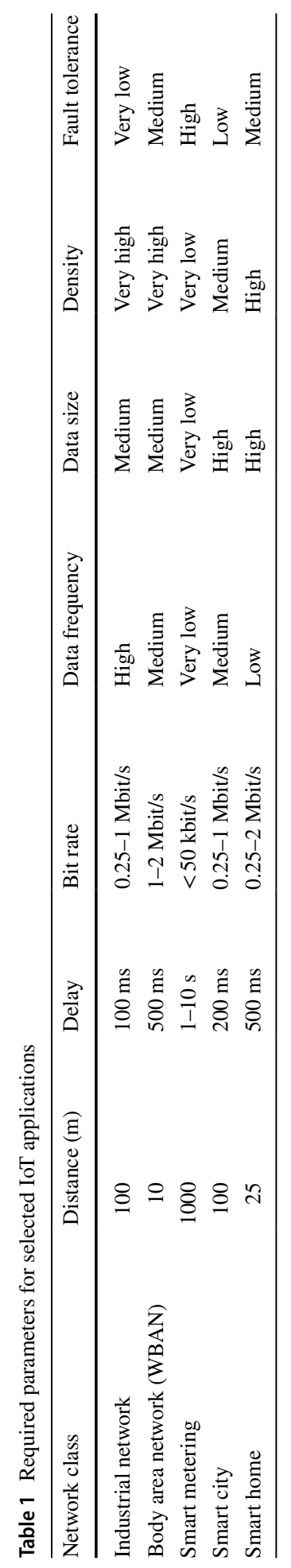


Table $2 \mathrm{P}_{\text {duty-cycle }}$ in body area network (2.4 GHz); synchronous (left), asynchronous (right)

\begin{tabular}{llllll}
\hline Synchronous & \multicolumn{5}{c}{ Asynchronous } \\
\hline$\Theta$ & 50 & $\mathrm{ppm}$ & $\Theta$ & 50 & $\mathrm{ppm}$ \\
$\mathrm{R}$ & 1 & $\mathrm{Mbit} / \mathrm{s}$ & $\mathrm{R}$ & 1 & $\mathrm{Mbit} / \mathrm{s}$ \\
$\mathrm{T}_{\text {s_data }}$ & 500 & $\mathrm{~ms}$ & $\mathrm{~T}_{\text {beacon }}$ & 500 & $\mathrm{~ms}$ \\
$\mathrm{t}_{\text {slot }}$ & 10 & $\mathrm{~ms}$ & $\mathrm{~T}_{\text {a_data }}$ & 5 & $\mathrm{~s}$ \\
$\mathrm{P}_{\text {duty-cycle }}$ & $\sim 0.7$ & $\mathrm{~mW}$ & $\mathrm{P}_{\text {duty-cycle }}$ & $\sim 0.8$ & $\mathrm{~mW}$ \\
\hline
\end{tabular}

\begin{tabular}{llllll}
\hline Synchronous & \multicolumn{5}{l}{ Asynchronous } \\
\hline$\Theta$ & 50 & $\mathrm{ppm}$ & $\Theta$ & 50 & $\mathrm{ppm}$ \\
$\mathrm{R}$ & 400 & $\mathrm{kbit} / \mathrm{s}$ & $\mathrm{R}$ & 400 & $\mathrm{kbit} / \mathrm{s}$ \\
$\mathrm{T}_{\text {s_data }}$ & 500 & $\mathrm{~ms}$ & $\mathrm{~T}_{\text {beacon }}$ & 500 & $\mathrm{~ms}$ \\
$\mathrm{t}_{\text {slot }}$ & 5 & $\mathrm{~ms}$ & $\mathrm{~T}_{\text {a_data }}$ & 10 & $\mathrm{~s}$ \\
$\mathrm{P}_{\text {duty-cycle }}$ & $\sim 0.4$ & $\mathrm{~mW}$ & $\mathrm{P}_{\text {duty-cycle }}$ & $\sim 0.6$ & $\mathrm{~mW}$ \\
\hline
\end{tabular}

Table $4 \mathrm{P}_{\text {duty-cycle }}$ in industrial network (2.4 GHz) (left), smart city application $(<1 \mathrm{GHz})$ (right)

\begin{tabular}{llllll}
\hline Synchronous & \multicolumn{5}{c}{ Synchronous } \\
\hline$\Theta$ & 50 & $\mathrm{ppm}$ & $\Theta$ & 50 & $\mathrm{ppm}$ \\
$\mathrm{R}$ & 400 & $\mathrm{kbit} / \mathrm{s}$ & $\mathrm{R}$ & 400 & $\mathrm{kbit} / \mathrm{s}$ \\
$\mathrm{T}_{\text {s_data }}$ & 100 & $\mathrm{~ms}$ & $\mathrm{~T}_{\text {s_data }}$ & 200 & $\mathrm{~ms}$ \\
$\mathrm{t}_{\text {slot }}$ & 10 & $\mathrm{~ms}$ & $\mathrm{t}_{\text {slot }}$ & 10 & $\mathrm{~ms}$ \\
$\mathrm{P}_{\text {duty-cycle }}$ & $\sim 3.1$ & $\mathrm{~mW}$ & $\mathrm{P}_{\text {duty-cycle }}$ & $\sim 1.4$ & $\mathrm{~mW}$ \\
\hline
\end{tabular}

Table $5 \mathrm{P}_{\text {duty-cycle }}$ in smart metering applications $(<1 \mathrm{GHz})$; synchronous (left), asynchronous (right)
Table $3 \mathrm{P}_{\text {duty-cycle }}$ in smart home applications $(<1 \mathrm{GHz})$; synchronous (left), asynchronous (right)

\begin{tabular}{lcllll}
\hline Synchronous & \multicolumn{5}{c}{ Asynchronous } \\
\hline$\Theta$ & 50 & $\mathrm{ppm}$ & $\Theta$ & 50 & $\mathrm{ppm}$ \\
$\mathrm{R}$ & 50 & $\mathrm{kbit} / \mathrm{s}$ & $\mathrm{R}$ & 50 & $\mathrm{kbit} / \mathrm{s}$ \\
$\mathrm{T}_{\text {s_data }}$ & 10 & $\mathrm{~s}$ & $\mathrm{~T}_{\text {beacon }}$ & 10 & $\mathrm{~s}$ \\
$\mathrm{t}_{\text {slot }}$ & 2 & $\mathrm{~ms}$ & $\mathrm{~T}_{\text {a_data }}$ & 3600 & $\mathrm{~s}$ \\
$\mathrm{P}_{\text {duty-cycle }}$ & $\sim 20$ & $\mu \mathrm{W}$ & $\mathrm{P}_{\text {duty-cycle }}$ & $\sim 60$ & $\mu \mathrm{W}$ \\
\hline
\end{tabular}

measurements, based on the datasheet of a similar CC1350 chip that works in both $<1 \mathrm{GHz}$ and $2.4 \mathrm{GHz}$ and belongs to the same $\mathrm{CC} 13 \mathrm{XX}$ device family. According to the documentation, the average current consumption of CC1350 in RX mode (reception) for the range $<1 \mathrm{GHz}$ is $5.4 \mathrm{~mA}$, while for the $2.4 \mathrm{GHz}$ range it is larger, equal to $6.4 \mathrm{~mA}$. The current consumption in the TX mode (transmitting) for the frequency range $<1 \mathrm{GHz}$ at $10 \mathrm{dBm}$ of the output power is $13.4 \mathrm{~mA}$ while for the $2.4 \mathrm{GHz}$ range at $9 \mathrm{dBm}$ it is $22.3 \mathrm{~mA}$. Based on the above-mentioned parameters, the correction value is equal to: $19 \%$ for $\mathrm{RX}$ mode and $66 \%$ for TX mode. This correction has been included in $\mathrm{E}_{\mathrm{IL}}, \mathrm{E}_{\mathrm{RX}}$ and $\mathrm{E}_{\mathrm{TX}}$ respectively, other parameters are the same as for the $<1 \mathrm{GHz}$ range. 
The smart home applications use the $<1 \mathrm{GHz}$ range. Due to the smaller data frequency, as well as their smaller size than in the case of body area network, the $t_{\text {slot }}$ time in synchronous mechanism was shortened twice, while the $\mathrm{T}_{\mathrm{a} \_ \text {data }}$ period in asynchronous mechanism was doubled. Smart home applications work at greater distances from the body area network and consume less energy for the duty-cycling mechanism. This causes the requirements for WuR to be more rigorous than in the previously discussed application, see Table 3.

It should be noted that when using asynchronous mechanism for the body area network and smart home, setting the correct $\mathrm{T}_{\text {beacon }}$ period is crucial to optimize energy consumption due to the relatively high data frequency. Beyond the optimum point, energy consumption is growing rapidly.

Most industrial wireless networks (IWN) use the $2.4 \mathrm{GHz}$ range, where more channels are available. The wider available spectrum allows using various spectrum spreading techniques, e.g. DSSS, FHSS, which allow to increase network efficiency. Due to the low expected node to node latency of 100-200 ms, the use of asynchronous duty-cycling mechanisms is not feasible, for such small $\mathrm{T}_{\text {beacon }}$ the energy consumption is growing very rapidly. Communication standards for IWN [1] such as ISA 100.11a, WirelessHART and other, use a synchronous mechanism (e.g. TDMA). In WirelessHART the duration of a time slot $\mathrm{t}_{\text {slot }}$ is fixed at $10 \mathrm{~ms}$, while in ISA100a.11a it is configurable and set to the specific value by the system manager when a device joins the network [40]. The situation is similar in smart city application where only the synchronous mechanism can be used due to the small required latency, see Table 4.

The smart metering applications are characterized by a very low data frequency and a large allowable latency, which allows using both techniques with good results, see Table 5. The very small amount of energy used for the duty-cycling mechanism and the large distance at which the nodes communicate, causes that WuR is not an alternative for these applications. The synchronous mechanism uses less energy than asynchronous one due to the very short time slot used ( $2 \mathrm{~ms}$ ), which corresponds only to $12 \mathrm{~B}$ at this bit rate.

We can also perform similar analysis looking for a balanced trade-off between delay reduction and power increase. In the presented comparison we have assumed typical node parameters. However, the proposed methodology is universal and can take into account other parameter values.

\section{Findings of the Study}

The main achievement of our research relates to the following issues:

- Identification of power critical points in duty-cycling communication schemes for synchronous and asynchronous techniques.

- Development of an original synchronization scheme for IoT networks targeted at reduction of power losses caused by the clock drift effect. This resulted in finding optimal beacon period in function of sender/receiver parameters, transmission speed and local clock inaccuracy.

- Development of universal power consumption models for representative IoT communication schemes (synchronous and asynchronous). They take into account diverse system parameters and provide capability of comparing and optimizing power consumption in IoT networks.

- Comparative analysis of scheduled duty-cycling and WuR approaches. 
The presented power consumption analysis methodology has been illustrated with a comparative study of power consumption for five classes of representative IoT networks. In particular, we have evaluated the power consumption for synchronous and asynchronous communication techniques and tried to point out situations where wake-up solution (WuR) is preferable. The presented study focused on performance comparison of traditional dutycycling techniques with an "on-demand" approach based on WuR. Taking into account only the elements of the protocol that are directly related to the duty-cycling mechanism, it is possible to compare different techniques without focusing on the specific MAC layer implementation. Derived equations are applicable for a wide range of transceivers. They are illustrated with calculations related to CC1310 TI chip.

WuR approach is a reasonable alternative for applications requiring small latency (time critical). When we use traditional duty-cycling techniques with the required node-to-node latencies equal to $100 \mathrm{~ms}$ and less, the node consumes typically at least $3.1 \mathrm{~mW}$ just on activities which are directly related to the used duty-cycling approach. Energy consumption of $1 \mathrm{~mW}$ for practical WuR implementations is currently possible to obtain at sensitivity of $-90 \mathrm{dBm}$. Hence, the use of WuR looks especially promising in industrial wireless networks (IWNs). These networks have gained interest in recent years in the Industry 4.0 concept. On the other hand, the use of WuR in applications where latency does not matter looks much less promising. A smart metering application is a special case, where $\mathrm{P}_{\text {duty_cycle }}$ less than $50 \mu \mathrm{W}$ can be easily achieved with traditional duty-cycling techniques in networks and where additionally nodes have to communicate on longer distances. Higher sensitivity of WuR strongly affects energy consumption, therefore achieving such low level of energy consumption by WuR while maintaining sensitivity at the required level is currently not possible in practical applications. Networks with short (single meters) or very short (single centimeters) communication distances are a particularly interesting group of WuR applications. In such applications, WuR can consume very small amount of energy by using passive or active architecture with low sensitivity.

\section{Conclusions}

This paper presents a taxonomy of duty-cycling schemes dedicated for IoT networks, including a detailed overview of energetic problems. Basing on this an original power consumption models have been proposed for synchronous and asynchronous communication techniques. These models take into account diverse parameters characterizing synchronization and transmission features combined with transmitter/receiver properties. The derived analytic formulas provide the capability of selecting optimal solutions for the designed networks (e.g. power consumption vs. transmission latency trade-off) as well as tuning communication protocol features. In addition, an original synchronization scheme was presented. This scheme takes into account the time elapsed since the last received synchronization beacon and adjusts the idle-listening time of the node. This results in additional energy savings.

The presented methodology along with derived models can be effectively used in adaptive MAC protocols to find an optimum trade-off between various MAC layer parameters in order to find an optimum energy consumption level. Further research is planned to use the presented approach for developing self-adaptive networks. In our research we will focus on industrial wireless networks (IWNs), the ISA100.11a standard will be used as a reference point for the developed adaptive layer 2 (L2). 
Acknowledgements This research was conducted as a part of a commercial research and development (R\&D) Project No. RPMA.01.02.00-14-9551/17-00 co-financed by the Mazovian Unit of EU Programs Implementation and European Union (EU) from the European Regional Development Fund (ERDF), priority axis 1 - "Increasing R\&D activities of enterprises in economy".

Open Access This article is distributed under the terms of the Creative Commons Attribution 4.0 International License (http://creativecommons.org/licenses/by/4.0/), which permits unrestricted use, distribution, and reproduction in any medium, provided you give appropriate credit to the original author(s) and the source, provide a link to the Creative Commons license, and indicate if changes were made.

\section{References}

1. Li, X., Li, D., Wan, J., Vasilakos, A. V., Lai, C. F., \& Wang, S. (2017). A review of industrial wireless networks in the context of Industry 4.0. Wireless Networks, 23(1), 23-41. https://doi. org/10.1007/s11276-015-1133-7.

2. Bomnale, A., \& Malgaonkar, S. (2018). Power optimization in wireless sensor networks. In 2018 International conference on communication information and computing technology (ICCICT) (pp. 1-6). New York: IEEE. https://doi.org/10.1109/iccict.2018.8325870.

3. Khan, J. A., Qureshi, H. K., \& Iqbal, A. (2015). Energy management in wireless sensor networks: A survey. Computers and Electrical Engineering, 41, 159-176. https://doi.org/10.1016/J.COMPE LECENG.2014.06.009.

4. Kim, B. S., Park, H. S., Kim, K. H., Godfrey, D., \& Kim, K. Il. (2017). A survey on real-time communications in wireless sensor networks. Wireless Communications and Mobile Computing. https:// doi.org/10.1155/2017/1864847.

5. Hu, J., Qian, Q., Fang, A., Wu, S., \& Xie, Y. (2016). Optimal data transmission strategy for healthcare-based wireless sensor networks: A stochastic differential game approach. Wireless Personal Communications, 89(4), 1295-1313. https://doi.org/10.1007/s11277-016-3316-7.

6. Lee, S. H., \& Choi, L. (2017). ZeroMAC: Toward a zero sleep delay and zero idle listening media access control protocol with ultralow power radio frequency wakeup sensor. International Journal of Distributed Sensor Networks, 13(8), 155014771771639. https://doi.org/10.1177/1550147717716397.

7. Polastre, J., Hill, J., \& Culler, D. (2004). Versatile low power media access for wireless sensor networks. In Proceedings of the 2nd international conference on embedded networked sensor systemsSenSys'04 (p. 95). New York: ACM. https://doi.org/10.1145/1031495.1031508.

8. Al-Anbagi, I., Erol-Kantarci, M., \& Mouftah, H. T. (2016). A survey on cross-layer quality-of-service approaches in WSNs for delay and reliability-aware applications. IEEE Communications Surveys and Tutorials, 18(1), 525-552. https://doi.org/10.1109/COMST.2014.2363950.

9. Doudou, M., Djenouri, D., \& Badache, N. (2013). Survey on latency issues of asynchronous MAC protocols in delay-sensitive wireless sensor networks. IEEE Communications Surveys and Tutorials, 15(2), 528-550. https://doi.org/10.1109/SURV.2012.040412.00075.

10. Zhuo, S., Wang, Z., Song, Y. Q., Wang, Z., \& Almeida, L. (2016). A traffic adaptive multi-channel MAC protocol with dynamic slot allocation for WSNs. IEEE Transactions on Mobile Computing, 15(7), 1600-1613. https://doi.org/10.1109/TMC.2015.2473852.

11. Schurgers, C., Tsiatsis, V., Ganeriwal, S., \& Srivastava, M. (2002). Optimizing sensor networks in the energy-latency-density design space. IEEE Transactions on Mobile Computing, 1(1), 70-80. https:// doi.org/10.1109/TMC.2002.1011060.

12. Soua, R., \& Minet, P. (2011). A survey on multichannel assignment protocols in wireless sensor networks. IFIP Wireless Days, 1(1), 1-3. https://doi.org/10.1109/WD.2011.6098201.

13. Suriyachai, P., Roedig, U., \& Scott, A. (2012). A survey of MAC protocols for mission-critical applications in wireless sensor networks. IEEE Communications Surveys and Tutorials, 14(2), 240-264. https ://doi.org/10.1109/SURV.2011.020211.00036.

14. Obeid, A. M., Atitallah, N., Loukil, K., Abid, M., \& Bensalah, M. (2018). A survey on efficient power consumption in adaptive wireless sensor networks. Wireless Personal Communications, 101(1), 101117. https://doi.org/10.1007/s11277-018-5678-5.

15. Di Valerio, V., Presti, F. L., Petrioli, C., Picari, L., \& Spaccini, D. (2016). A self-adaptive protocol stack for underwater wireless sensor networks. In OCEANS 2016-Shanghai. https://doi.org/10.1109/ oceansap.2016.7485478. 
16. Kozłowski, A., \& Sosnowski, J. (2018). Evaluating energy consumption in wireless sensor networks applications. In Proceedings of SPIE, photonics applications in astronomy, communications, industry, and high energy physics experiments.

17. Singh, S. K., Kumar, P., \& Singh, J. P. (2018). An energy efficient protocol to mitigate hot spot problem using unequal clustering in WSN. Wireless Personal Communications, 101(2), 799-827. https:// doi.org/10.1007/s11277-018-5716-3.

18. Harold Robinson, Y., Golden Julie, E., Balaji, S., \& Ayyasamy, A. (2017). Energy aware clustering scheme in wireless sensor network using neuro-fuzzy approach. Wireless Personal Communications, 95(2), 703-721. https://doi.org/10.1007/s11277-016-3793-8.

19. Mishra, R., Jha, V., Tripathi, R. K., \& Sharma, A. K. (2017). Energy efficient approach in wireless sensor networks using game theoretic approach and ant colony optimization. Wireless Personal Communications, 95(3), 3333-3355. https://doi.org/10.1007/s11277-017-4000-2.

20. Pantazis, N. A., Nikolidakis, S. A., \& Vergados, D. D. (2013). Energy-efficient routing protocols in wireless sensor networks: A survey. IEEE Communications Surveys and Tutorials, 15(2), 551-591. https://doi.org/10.1109/SURV.2012.062612.00084.

21. Liu, F., Wang, Y., Lin, M., Liu, K., \& Wu, D. (2017). A distributed routing algorithm for data collection in low-duty-cycle wireless sensor networks. IEEE Internet of Things Journal, 4(5), 1420-1433. https://doi.org/10.1109/JIOT.2017.2734280.

22. Dutta, P., Dawson-Haggerty, S., Chen, Y., Liang, C.-J. M., \& Terzis, A. (2010). Design and evaluation of a versatile and efficient receiver-initiated link layer for low-power wireless. In Proceedings of the 8th ACM conference on embedded networked sensor systems-SenSys'10 (pp. 1-14). New York: ACM. https://doi.org/10.1145/1869983.1869985.

23. Sun, Y., Gurewitz, O., \& Johnson, D. B. (2008). RI-MAC: A receiver-initiated asynchronous duty cycle MAC protocol for dynamic traffic loads in wireless sensor networks. In Proceedings of the 6th ACM conference on embedded network sensor systems-SenSys'08, 81 LNICST, 1. https://doi. org/10.1145/1460412.1460414.

24. Buettner, M., Yee, G. V., Anderson, E., \& Han, R. (2006). X-MAC: A short preamble MAC protocol for duty-cycled wireless sensor networks. In Proceedings of the 4th international conference on embedded networked sensor systems-SenSys'06 (pp. 307-320). New York: ACM. https://doi. org/10.1145/1182807.1182838.

25. Carrano, R. C., Passos, D., Magalhaes, L. C. S., \& Albuquerque, C. V. N. (2014). Survey and taxonomy of duty cycling mechanisms in wireless sensor networks. IEEE Communications Surveys and Tutorials, 16(1), 181-194. https://doi.org/10.1109/SURV.2013.052213.00116.

26. Huang, P., Xiao, L., Soltani, S., Mutka, M. W., \& Xi, N. (2013). The evolution of MAC protocols in wireless sensor networks: A survey. IEEE Communications Surveys and Tutorials, 15(1), 101-120. https://doi.org/10.1109/SURV.2012.040412.00105.

27. Blanckenstein, J., Klaue, J., \& Karl, H. (2015). A survey of low-power transceivers and their applications. IEEE Circuits and Systems Magazine, 15(3), 6-17. https://doi.org/10.1109/MCAS.2015.2450634.

28. Piyare, R., Murphy, A. L., Kiraly, C., Tosato, P., \& Brunelli, D. (2017). Ultra low power wake-up radios: A hardware and networking survey. IEEE Communications Surveys and Tutorials, 19(4), 21172157. https://doi.org/10.1109/COMST.2017.2728092.

29. Huang, H., Yun, J., Zhong, Z., Kim, S., \& He, T. (2013). PSR: Practical synchronous rendezvous in low-duty-cycle wireless networks. In 2013 proceedings IEEE INFOCOM (pp. 2661-2669). New York: IEEE. https://doi.org/10.1109/infcom.2013.6567074.

30. Lin, E.-Y. A., Rabaey, J. M., \& Wolisz, A. (2004). Power-efficient rendez-vous schemes for dense wireless sensor networks. In 2004 IEEE international conference on communications (IEEE Cat. No.04CH37577) (Vol. 7, pp. 3769-3776). New York: IEEE. https://doi.org/10.1109/icc.2004.1313259.

31. Oller, J., Demirkol, I., Casademont, J., Paradells, J., Gamm, G. U., \& Reindl, L. (2016). Has time come to switch from duty-cycled MAC protocols to wake-up radio for wireless sensor networks? IEEE/ACM Transactions on Networking, 24(2), 674-687. https://doi.org/10.1109/TNET.2014.2387314.

32. Heurtefeux, K., \& Valois, F. (2012). Is RSSI a good choice for localization in wireless sensor network? In 2012 IEEE 26th international conference on advanced information networking and applications (pp. 732-739). New York: IEEE. https://doi.org/10.1109/aina.2012.19.

33. Wu, Y.-C., Chaudhari, Q., \& Serpedin, E. (2011). Clock synchronization of wireless sensor networks. IEEE Signal Processing Magazine, 28(1), 124-138. https://doi.org/10.1109/MSP.2010.938757.

34. Stanislowski, D., Vilajosana, X., Wang, Q., Watteyne, T., \& Pister, K. S. J. (2014). Adaptive synchronization in IEEE802.15.4e networks. IEEE Transactions on Industrial Informatics, 10(1), 795-802. https://doi.org/10.1109/tii.2013.2255062.

35. Kim, R., Nagayama, T., Jo, H., Spencer, J., \& Spencer, Jr., B. F. (2012). Preliminary study of lowcost GPS receivers for time synchronization of wireless sensors. In Proceedings of SPIE sensors 
and smart structures technologies for civil mechanical and aerospace systems (Vol. 8345(1), pp. 83451A-83451A-9). https://doi.org/10.1117/12.915394.

36. Li, L., Xing, G., Sun, L., Huangfu, W., Zhou, R., \& Zhu, H. (2011). Exploiting FM radio data system for adaptive clock calibration in sensor networks. In Proceedings of the 9th international conference on mobile systems, applications, and services-MobiSys'11 (p. 169). New York: ACM. https://doi. org/10.1145/1999995.2000012.

37. Engeler, D. (2012). Performance analysis and receiver architectures of DCF77 radio-controlled clocks. IEEE Transactions on Ultrasonics, Ferroelectrics, and Frequency Control, 59(5), 869-884. https://doi. org/10.1109/TUFFC.2012.2272.

38. Macii, D., Ageev, A., \& Somov, A. (2009, May). Power consumption reduction in wireless sensor networks through optimal synchronization. In 2009 IEEE instrumentation and measurement technology conference (pp. 1346-1351). https://doi.org/10.1109/imtc.2009.5168665.

39. Lee, T.-H., Chu, H.-C., Chang, L.-H., Chiang, H.-S., \& Lin, Y.-W. (2013). Modeling and performance analysis of route-over and mesh-under routing schemes in 6LoWPAN under error-prone channel condition. Journal of Applied Mathematics, 2013, 1-9. https://doi.org/10.1155/2013/242483.

40. Petersen, S., \& Carlsen, S. (2011). WirelessHART versus ISA100.11a: The format war hits the factory floor. IEEE Industrial Electronics Magazine, 5(4), 23-34. https://doi.org/10.1109/mie.2011.943023.

Publisher's Note Springer Nature remains neutral with regard to jurisdictional claims in published maps and institutional affiliations.
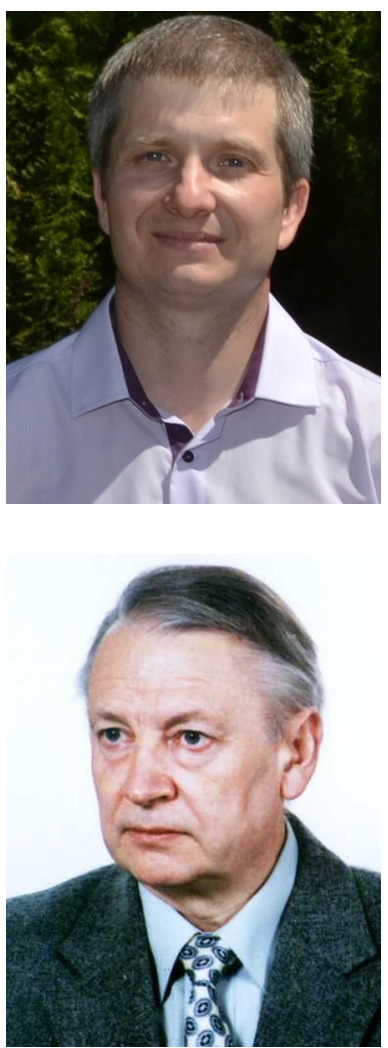

Adam Kozłowski received M.Sc. degree at the Warsaw University of Technology in 2002. Currently, he is working on a Ph.D. at the same university, Institute of Computer Science. He is a co-owner and president of a software development company for over decade. He is involved in many commercial and research projects. He is a research leader in the R\&D Project No. RPMA.01.02.00-14-9551/17-00 cofinanced by EU. He cooperates with the Institute of Computer Science and his research interests include software engineering, wireless industrial networks, network and protocol optimization mainly in the Industry 4.0 field.

Janusz Sosnowski is a full professor at the Warsaw University of Technology, Institute of Computer Science. He has published more than 200 papers and several books and has participated in many scientific and industrial projects (mostly as project coordinator). His research interests include dependable computing, system architecture and software engineering issues. He is well known in computer science society, senior member of IEEE, PC member of many international conferences. 